\title{
Propuesta de actualización de saberes en campos numéricos para estudiantes de pedagogía en telesecundaria
}

\author{
Proposal for Updating Knowledge in Numerical Fields for Students of \\ Pedagogy in Telesecundaria
}

\section{Proposta de atualização de conhecimentos em campos numéricos para estudantes de pedagogia em telesecundaria}

Enrique Gómez Segura Escuela Normal Urbana Federal Prof. Rafael Ramírez, México egos72@hotmail.com https://orcid.org/0000-0002-3730-4556

\begin{abstract}
Resumen
El presente artículo muestra una secuencia didáctica dirigida a los estudiantes de la licenciatura en Enseñanza y Aprendizaje en Telesecundaria de la Escuela Normal Urbana Federal Prof. Rafael Ramírez de Chilpancingo, Guerrero, con la finalidad de sistematizar sus conocimientos en los diversos campos numéricos. El objetivo es conocer y manejar la clasificación de los números reales, porque es de suma importancia para los docentes en formación: si saben las características de cada uno de ellos, podrán realizar las operaciones adecuadas, sin confundirse entre los diversos campos numéricos. El presente trabajo se fundamenta en la teoría de David Ausubel (Méndez, 1993), que trata sobre el aprendizaje significativo. Por otra parte, es muy común que los estudiantes de secundaria confundan las reglas de operación que se aplican en la suma y resta, y las utilicen para multiplicar y dividir; esto tiene una explicación, los conocimientos matemáticos son como una espiral, cada vez se van incrementando y las reglas van cambiando, por eso cada campo numérico nuevo
\end{abstract}




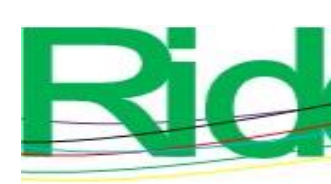

Revista Iberoamericana para la
Investigación y el Desarrollo Educativo
ISSN $2007-7467$

representa para el alumno un conflicto cognitivo, de tal manera que no es la misma regla sumar dos números positivos que sumar dos fracciones que tienen diferente denominador. El resultado de la suma de dos números enteros positivos también es positivo y es mayor que cualquier sumando; mientras que la suma de las fracciones tiene otro procedimiento muy distinto; en este caso, se ocupó el mínimo común múltiplo y las fracciones equivalentes para calcular la suma. Lo mismo sucede con los demás campos numéricos.

Palabras clave: actualización docente, números reales, operaciones básicas, secuencia didáctica, telesecundaria.

\section{Abstract}

The present article shows a didactic sequence directed to the students of the degree in Teaching and Learning in Telesecundaria of the Escuela Normal Urbana Federal Prof. Rafael Ramírez of the city of Chilpancingo, Guerrero, with the purpose of systematizing their knowledge in the various numerical fields. The objective is to know and manage the classification of real numbers, because it is of utmost importance for teachers in training: if they know the characteristics of each one, they will be able to carry out the appropriate operations, without being confused between the various numerical fields. The present work is based on the theory of David Ausubel (Méndez, 1993), which deals with meaningful learning. Moreover, it is very common for high school students to confuse the rules of operation that apply to addition and subtraction, and use them to multiply and divide; this has an explanation, mathematical knowledge is like a spiral: each time it increases and the rules change, so each new number field represents a cognitive conflict for the student, in such a way that adding two numbers is not the same rule positives that add two fractions that have a different denominator. The result of the sum of two positive integers is also positive and is greater than any addend; while the sum of the fractions has another, very different procedure; in this case, the least common multiple and the equivalent fractions were used to calculate the sum. The same is true for the other number fields.

Keywords: teaching update, real numbers, basic operations, didactic sequence, telesecundaria. 


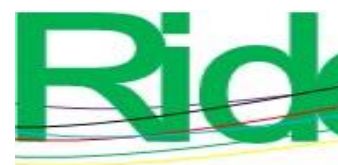
Revista Iberoamericana para la
Investigación y el Desarrollo Educativo
ISSN $2007-7467$

\section{Resumo}

Este artigo apresenta uma sequência didática direcionada aos alunos da graduação em Ensino e Aprendizagem em Telesecundaria da Escuela Normal Urbana Federal, Prof. Rafael Ramírez de Chilpancingo, Guerrero, com o objetivo de sistematizar seus conhecimentos nas diversas áreas numéricas. O objetivo é conhecer e gerenciar a classificação de números reais, pois é de extrema importância para os professores em formação: se eles conhecerem as características de cada um deles, serão capazes de realizar as operações apropriadas, sem serem confundidos entre os vários campos numéricos. O presente trabalho é baseado na teoria de David Ausubel (Méndez, 1993), que trata da aprendizagem significativa. Por outro lado, é muito comum que estudantes do ensino médio confundam as regras de operação que se aplicam à adição e subtração e as usam para multiplicar e dividir; isso tem uma explicação, o conhecimento matemático é como uma espiral, cada vez que eles aumentam e as regras mudam; portanto, cada novo campo numérico representa um conflito cognitivo para o aluno, de forma que a adição de dois números não é a mesma regra positivos que adicionam duas frações que têm um denominador diferente. $\mathrm{O}$ resultado da soma de dois números inteiros positivos também é positivo e é maior que qualquer adendo; enquanto a soma das frações tem outro procedimento muito diferente; neste caso, o mínimo múltiplo comum e as frações equivalentes foram usadas para calcular a soma. O mesmo vale para os outros campos numéricos.

Palavras-chave: atualização de ensino, números reais, operações básicas, sequência didática, telesecundaria. 


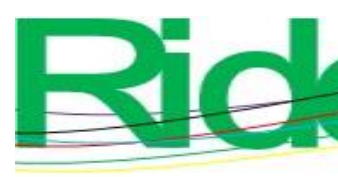

Revista Iberoamericana para la Investigación y el Desarrollo Educativo ISSN $2007-7467$

\section{Introducción}

Todos los profesores que imparten la asignatura de matemáticas tienen la obligación de conocer la clasificación de los números reales con la finalidad de realizar adecuadamente las operaciones, porque cada campo numérico tiene sus propias reglas de operación.

En términos reales, encontramos que los estudiantes de recién ingreso a la licenciatura de Enseñanza y Aprendizaje en Telesecundaria de la Escuela Normal Urbana Federal Prof. Rafael Ramírez de Chilpancingo, Guerrero, México, cuentan con bajos niveles de conocimientos matemáticos. Esto representa un reto para formar buenos docentes, por eso se hacen trabajos documentales y de campo que permitan a los profesores normalistas subsanar esas irregularidades en el alumnado.

Por lo anterior, se diseñó una secuencia didáctica que les permitiera a los estudiantes analizar colectiva e individualmente los problemas numéricos, realizar reflexiones y conclusiones para una mejor comprensión.

El trabajo colectivo permite conocer los diversos puntos de vista de otros compañeros para resolver problemas. Las ideas pueden ser buenas o no, por eso se analizan colectivamente, para institucionalizar el conocimiento, todo esto se realiza bajo la supervisión del docente, quien tiene la función de mediador.

El presente artículo describe la evolución de los campos numéricos. En algunas ocasiones se parafrasea el video de Sáenz (2019), pero también se toma en cuenta la historia de las matemáticas, tal y como lo plantea Struik (1998). Se inicia con los números naturales, los cuales fueron los primeros que se crearon por la humanidad, después con los enteros, pasando a los racionales y concluyendo con los irracionales. El paso de cada uno de ellos se ve con la dificultad de una operación que no puede ser resuelta con el campo numérico analizado hasta ese momento; además, se van presentando por conjunto y en recta numérica. Con esta última se pretende que sea más explícito lo que se construye.

\section{Metodología}

El desarrollo de este trabajo se llevó a cabo de la siguiente manera:

a) Se diseñó la secuencia didáctica de los números reales.

b) Se presentó el conjunto de los números naturales con algunas operaciones que no pueden dar resultados en ese campo numérico. 

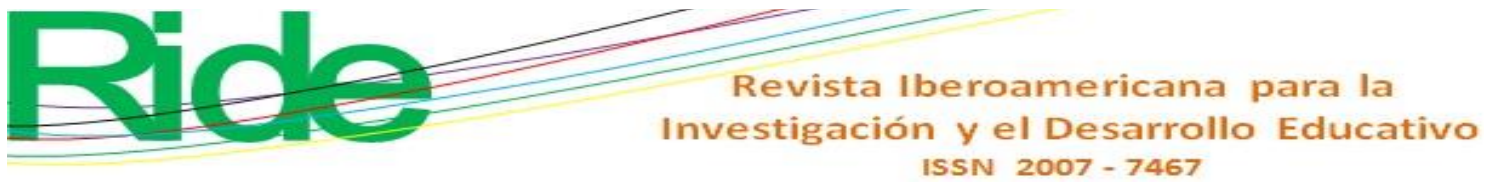

c) De la misma manera se hizo con los demás números: enteros, racionales e irracionales.

d) En cada campo se analizaron las reglas de operación y realizaron los ejercicios correspondientes, para familiarizarse y construir sus propias definiciones.

\section{Desarrollo de las actividades}

- Escribe los números que conoces o has ocupado:

- ¿Cómo se llaman?

La comunidad matemática ha establecido una clasificación de los números que a continuación se presentan con algunas actividades de reforzamiento y reflexión. Las conclusiones colectivas e individuales son importantes que se realicen al término de cada actividad, así como al finalizar el campo numérico analizado. Es importante ponerse de acuerdo con el grupo para institucionalizar el conocimiento que se está aprendiendo.

¡Comenzamos!

\section{La clasificación de los números reales Los números naturales}

Figura 1. Los números en los pueblos primitivos

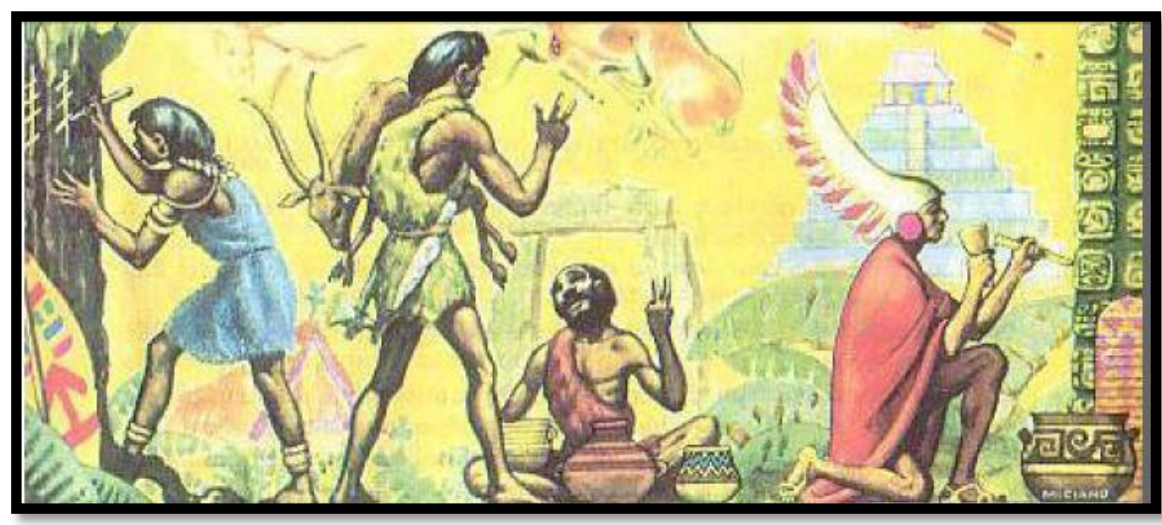

Fuente: Baldor (1982)

Históricamente, el hombre ha contado por necesidad. Sus registros se encuentran en diversos materiales. Uno de los más antiguos es el hueso de Lebombo (son huesos de 


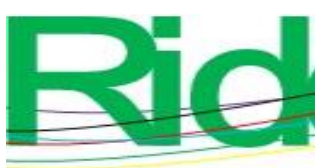

Revista Iberoamericana para la
Investigación y el Desarrollo Educativo
ISSN $2007-7467$

babuino), según Sánchez (2 de febrero de 2014), el cual tiene 28 marcas, por lo que se puede relacionar con el ciclo lunar o menstrual.

Figura 2. Hueso de Lebombo

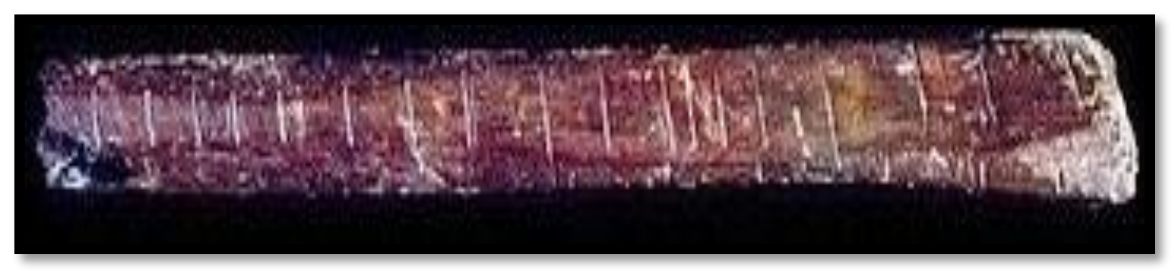

Fuente: Sánchez (2 de febrero de 2014)

Figura 3. Babuinos

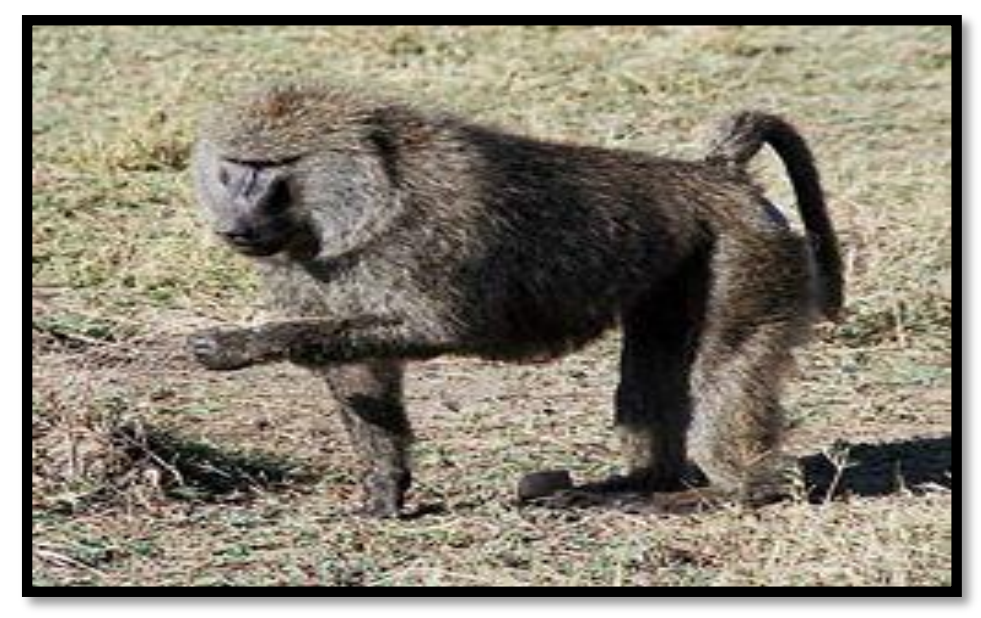

Fuente: Wikipedia (26 de noviembre de 2019)

El hueso de Lebombo es un instrumento para contar el tiempo de algún ciclo natural; tiene una antigüedad de 35000 años y fue hallado en Border Cave, en las montañas de Lebombo, entre Sudáfrica y Suazilandia (Sánchez, 2 de febrero de 2014).

De acuerdo con el canal MateFacil (8 de agosto de 2016), los primeros números se llaman naturales y se representan con una $N$ mayúscula con doble raya $(\mathbb{N})$. El hombre, al contar, contaba lo que veía en la naturaleza, lo que no veía no lo contaba, por eso fue difícil para muchas culturas la invención del cero. Bajo esta perspectiva, el primer número es el uno y no el cero. 

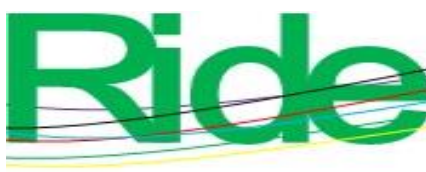

Revista Iberoamericana para la Investigación y el Desarrollo Educativo ISSN 2007 - 7467

En una recta numérica se representa de la siguiente forma (ver figura 4).

Figura 4. Números naturales

\begin{tabular}{|c|c|c|c|c|c|c|c|c|c|c|c|}
\hline 1 & 2 & 3 & 4 & 5 & 6 & 7 & 8 & 9 & 10 & 11 & 12 \\
\hline
\end{tabular}

Fuente: Elaboración propia

Con los números naturales se pueden hacer diversas operaciones aritméticas, como la suma, resta, multiplicación y división.

Tabla 1. Sumas de números Naturales

\section{Realiza las siguientes sumas}

a) $23+45=$

b) $34+78=$

c) $103+59=$

Fuente: Elaboración propia

Contesta las siguientes preguntas

- ¿ ¿Todos los resultados son números naturales?

- ¿Se requieren otros tipos de números fuera de los naturales?

Tabla 2. Restas de números Naturales

\section{Realiza las siguientes restas}

a) $150-60=$

b) $20-20=$

c) $54-100=$

\section{Fuente: Elaboración propia}

Contesta las siguientes preguntas

- ¿ ¿Todos los resultados son números naturales?

- ¿Entonces se requieren otros tipos de números fuera de los naturales?

Conforme la sociedad se fue desarrollando, el primer campo numérico fue insuficiente para resolver problemas, por eso fue necesario crear otro campo numérico. 
Tabla 3. Multiplicación de números Naturales

\section{Realiza las siguientes multiplicaciones}

a) $15 \times 8=$

b) $23 \times 12=$

c) $189 \times 45=$

\section{Fuente: Elaboración propia}

Contesta las siguientes preguntas

- ¿ ¿Todos los resultados son números naturales?

- ¿Se requieren otros tipos de números fuera de los naturales?

Realiza las siguientes divisiones
a) $\frac{15}{3}=$
b) $\frac{60}{5}=$
c) $\frac{25}{6}=$

Contesta las siguientes preguntas

- ¿ ¿Todos los resultados son números naturales?

- ¿Se requieren otros tipos de números fuera de los naturales?

Contestando las interrogantes, nos damos cuenta de que los resultados de la suma siempre son números naturales. Para esta operación no hay problemas para crear otro campo numérico; sin embargo, para las operaciones de la resta, hay un obstáculo por resolver: si el primer número (minuendo) es menor que el segundo número (sustraendo), el resultado (diferencia) no se encuentra ubicado dentro de los números naturales, por lo tanto, se hacía indispensable crear otro campo numérico.

Para el caso de las operaciones de multiplicación, no hay ningún problema, todos los resultados son números naturales; pero en el caso de la división, hay ciertos resultados que no pertenecen al campo de los números naturales, por ejemplo: $\frac{1}{3}, \frac{-2}{8}$, etc. 


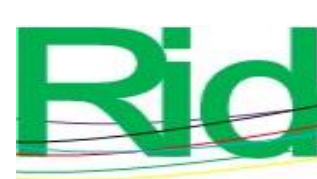

Revista Iberoamericana para la Investigación y el Desarrollo Educativo ISSN 2007-7467

\section{Los números enteros}

Figura 5. Ferias medievales

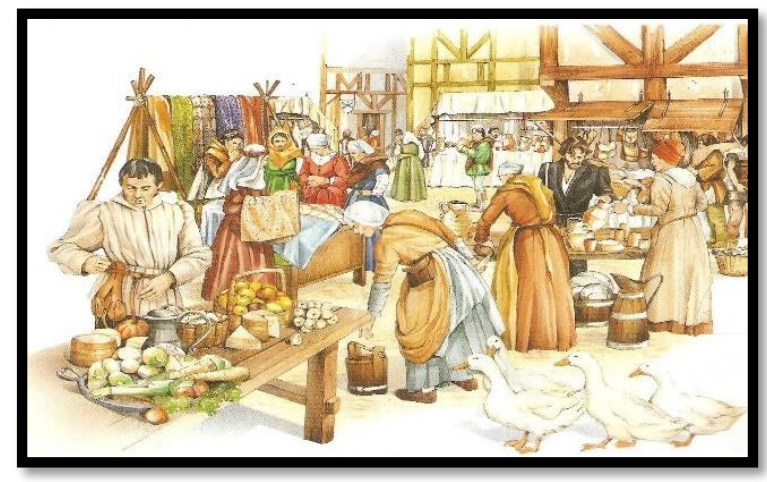

Fuente: http://www.escuelapedia.com/ferias-medievales/

Siguiendo a Wikipedia (19 de julio de 2020), cuando el comercio aparece en la sociedad, se hizo necesario representar numéricamente diversas situaciones, por ejemplo: cuando toda la mercancía se había vendido (el cero), las ganancias de una determinada venta (números positivos) y pérdidas obtenidas por diversos motivos (números negativos). Esto originó el campo numérico de los números enteros, los cuales se representan con el símbolo $\mathbb{Z}$, y matemáticamente se escribe de la siguiente manera:

$$
\mathbb{Z}=\{\ldots,-3,-2,-1,0,1,2,3, \ldots\}
$$

Los tres puntos significan 'y así sucesivamente'.

Los números enteros representados en la recta numérica se observan en la figura 6.

Figura 6. La recta numérica

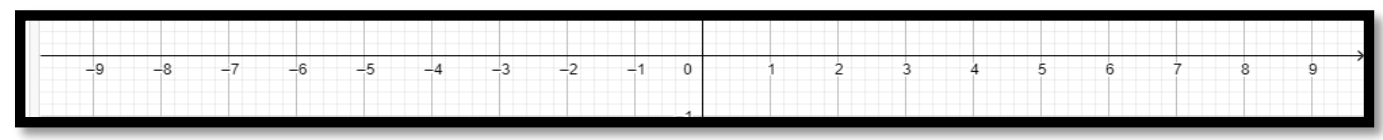

Fuente: Elaboración propia

Con la creación de este campo numérico se resuelve el problema de la resta que se había planteado anteriormente: $54-100=-46$. En términos prácticos, el problema pudo haberse planteado de la siguiente manera: "E1 Sr. Carlos tenía \$54.00 y abonó esa cantidad a 


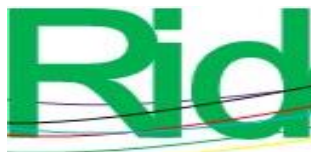

Resuelve las siguientes sumas

a) $3+5=$

Procedimiento:

1) Se ubica al número tres en la recta numérica

2) Se desplaza cinco unidades a la derecha porque el cinco es positivo.

Figura 7. Suma de dos números positivos

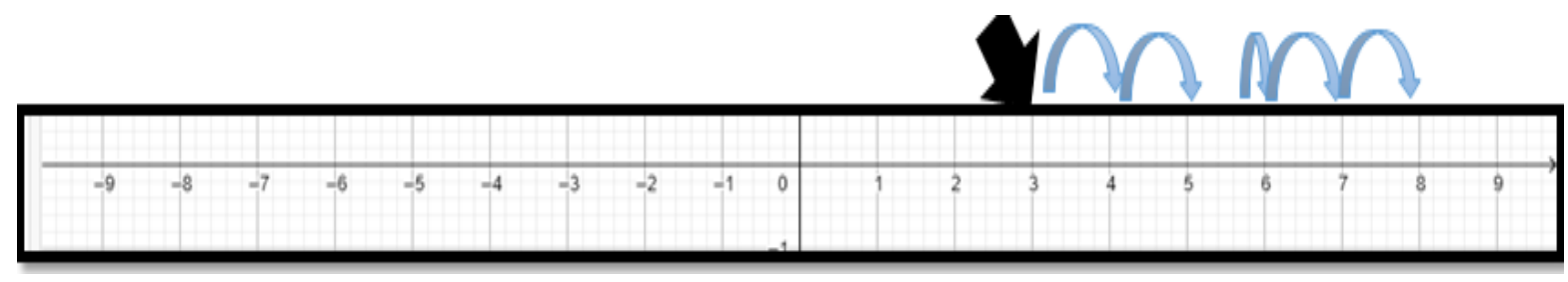

Fuente: Elaboración propia

El resultado es +8

b) $-1-3=$

Procedimiento:

1) Se ubica al número -1 en la recta numérica.

2) Se desplaza tres unidades a la izquierda porque el tres es negativo.

Figura 8. Suma de dos números negativos

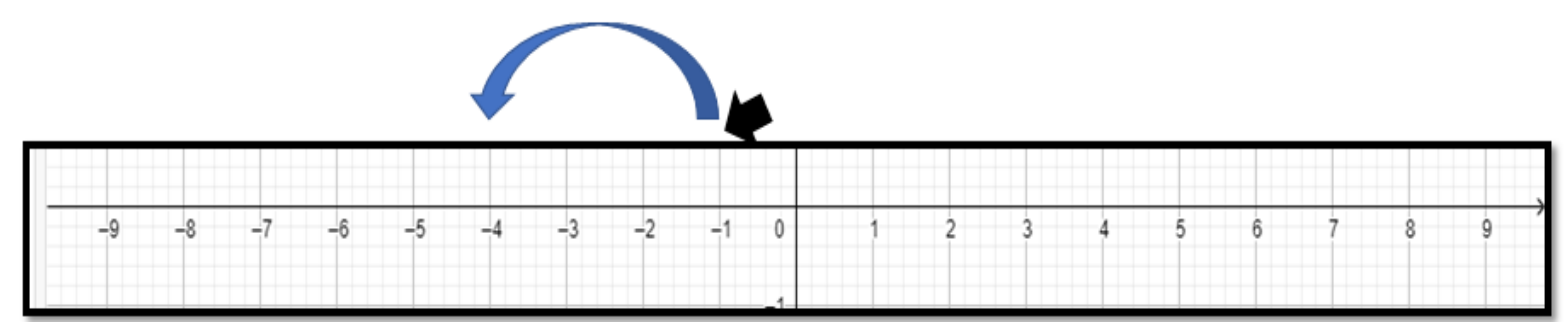

Fuente: Elaboración propia

El resultado es -4 .

Reflexión: si sumamos (simplificar a un solo número) dos números de signos iguales, la operación es una suma. El resultado tendrá el signo de los sumandos (positivo o negativo), según sea el caso. 
Tabla 6. Suma de números con igual signo

Caso I: Cuando ambos números tienen el mismo signo, la operación es una suma; el signo es positivo o negativo según sea el caso.

Ejemplos:

\begin{tabular}{|l|l|l|l|}
\hline a) $2+3=+5$ & b) $7+2=+9$ & c) $4+6=+10$ & d) $12+14=+26$ \\
\hline e) $-2-3=-5$ & f) $-7-2=-9$ & g) $-4-6=-10$ & h) $-12-14=-26$ \\
\hline
\end{tabular}

\section{Resuelve las siguientes sumas en tu cuaderno}

\begin{tabular}{|l|l|l|l|}
\hline a) $6+3=$ & b) $12+5=$ & c) $2+9=$ & d) $12+8=$ \\
\hline e) $15+7=$ & f) $17+5=$ & g) $11+3=$ & h) $14+2=$ \\
\hline i) $24+11=$ & j) $17+12=$ & k) $27+18=$ & 1) $32+45=$ \\
\hline m) $-2-8=$ & n) $-12-6=$ & ñ $-11-10=$ & o) $-7-9=$ \\
\hline p) $-12-25=$ & q) $-8-15=$ & r) $-9-12=$ & s) $-22-11=$ \\
\hline t) $-17-6=$ & u) $-9-11=$ & v) $-27-17=$ & w) $-42-35=$ \\
\hline
\end{tabular}

Fuente. Elaboración propia

En la suma de dos números que tienen diferente signo:

- $\quad$ Si es número positivo, se desplazará hacia la derecha.

- $\quad$ Si es negativo, se desplazará hacia la izquierda.

Resuelve la siguientes sumas

a) $-3+5=$

Procedimiento:

1) Se ubica al número -3 en la recta numérica

2) Se desplaza cinco unidades a la derecha porque el cinco es positivo.

Figura 9. Suma de dos números de diferente signo

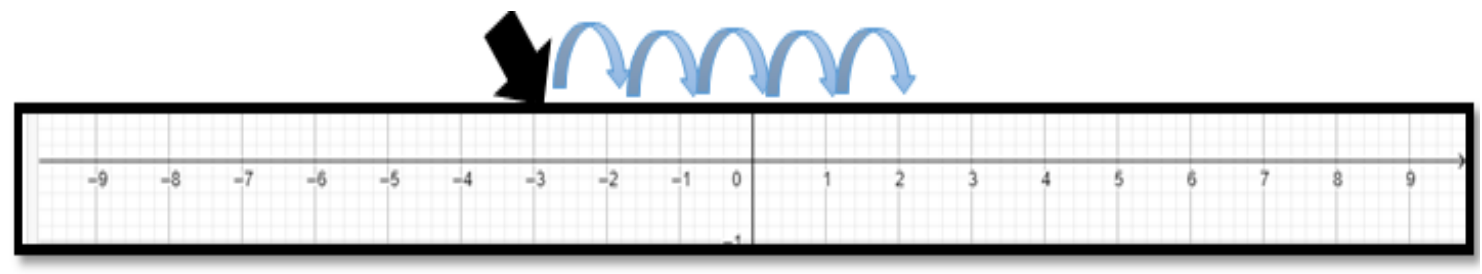

Fuente: Elaboración propia 
El resultado es +2

b) $4-6=$

Procedimiento:

1) Se ubica al número cuatro en la recta numérica.

2) Se desplaza seis unidades a la izquierda porque el seis es negativo.

Figura 10. Cuando el número negativo es mayor que el positivo

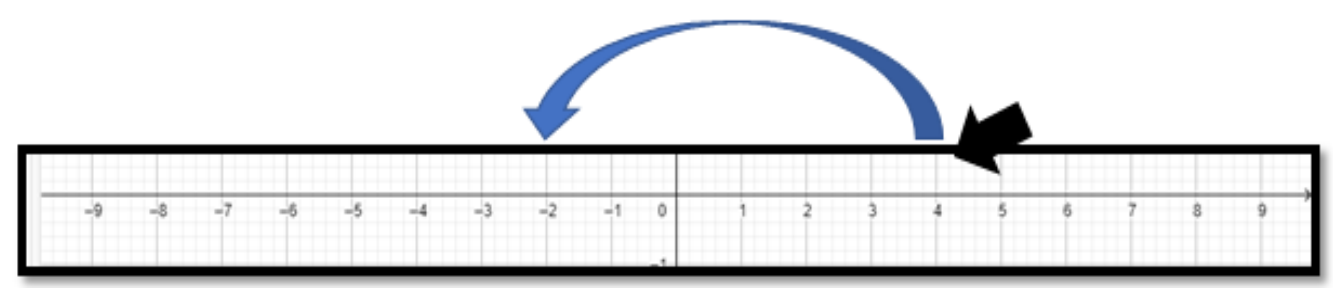

Fuente: Elaboración propia

El resultado es - 2

Reflexión: si sumamos (simplificar a un solo número) dos números de diferente signo, la operación es una resta. Tomando los números con valor absoluto, se resta al número mayor el menor, y queda el signo del mayor.

Los siguientes ejercicios pueden ayudar comprender mejor las operaciones (ver tabla 4).

Tabla 7. Suma de números con diferente signo

Caso II: Cuando los números tienen diferente signo, la operación es una resta, es decir, al número de mayor absoluto se le resta el de menor valor absoluto, quedando el signo en el resultado el de mayor valor absoluto.

Ejemplos:

\begin{tabular}{|l|l|l|l|}
\hline a) $2-6=-4$ & b) $4-10=-6$ & c) $6-16=-10$ & d) $8-12=-4$ \\
\hline e) $-25+10=-15$ & f) $-17+27=10$ & g) $-22+34=+12$ & h) $-23+15=-8$ \\
\hline \multicolumn{2}{|l|}{ Resuelve las siguientes operaciones en tu cuaderno } \\
\hline a) $5-9=$ & b) $3-12=$ & c) $7-15=$ & d) $9-17=$ \\
\hline e) $12-23=$ & f) $28-23=$ & g) $39-25=$ & h) $12-32=$ \\
\hline i) $-19+25=$ & j) $-31+25=$ & k) $-22+34=$ & l) $32-24=$ \\
\hline
\end{tabular}

Fuente. Elaboración propia 

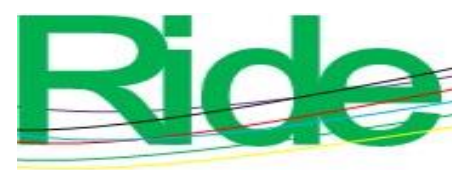

Cada nuevo conocimiento representa un conflicto cognitivo para el alumnado, el cual, si es superado, puede adquirir los siguientes niveles escolares (temas de mayor grado de complejidad), los cuales cada vez requieren de mayores bases matemáticas para su dominio.

\section{Interpretación de las fracciones}

De acuerdo con Tu profe en línea (13 de mayo de 2015), la fracciones comunes son aquellas que tienen a un numerador y un denominador, por ejemplo:

$$
\frac{3}{8}=\frac{\leftarrow \quad \text { Numerador }}{\leftarrow \text { Denominador }}=\frac{\text { indica las partes que se toma de la unidad }}{\text { indica las partes en que se divide la unidad }}
$$

\section{Representación de las fracciones}

Escribe la fracción que le corresponda a la parte coloreada

Figura 12. Juegos con fracciones: lectura, comparación, fracciones

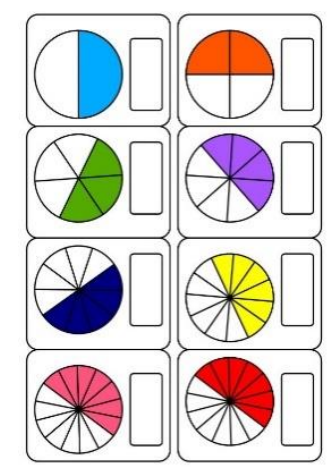

Fuente: Rodríguez (s. f.)

Contesta las siguientes interrogantes

1) ¿Cómo son las fracciones?

2) ¿Cómo puedes comprobar?

\section{Operaciones de las fracciones}

Las siguientes actividades retoman el video de "Comprendiendo la suma y resta de fracciones" (Matemática profe Alex, 28 de octubre de 2018):

- Suma de fracciones que tienen el mismo denominador: 


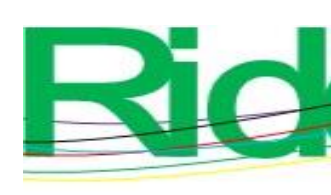

Revista Iberoamericana para la Investigación y el Desarrollo Educativo ISSN 2007 - 7467
a) $\frac{6}{4}+\frac{7}{4}=\frac{13}{4}$
b) $\frac{5}{7}+\frac{12}{7}=\frac{17}{7}$
c) $\frac{14}{12}-\frac{6}{12}=\frac{8}{12}=\frac{4}{6}=\frac{2}{3}$
d) $\frac{12}{8}-\frac{13}{8}-\frac{5}{8}=\frac{-6}{8}=-\frac{3}{4}$

Resuelve las siguientes sumas de fracciones

Tabla 8. Suma de fracciones homogéneas

\begin{tabular}{|l|l|l|l|}
\hline 1) $\frac{5}{3}+\frac{12}{3}=$ & 2) $\frac{7}{5}+\frac{8}{5}=$ & 3) $\frac{5}{9}+\frac{14}{9}=$ & 4) $\frac{4}{14}+\frac{10}{14}=$ \\
\hline 5) $\frac{15}{7}+\frac{13}{7}=$ & 6) $\frac{17}{8}+\frac{18}{8}=$ & 7) $\frac{28}{3}+\frac{14}{3}-\frac{7}{3}=$ & 8) $\frac{43}{4}+\frac{10}{4}-\frac{20}{4}=$ \\
\hline 9) $\frac{12}{7}-\frac{53}{7}=$ & 10) $\frac{19}{8}+\frac{14}{8}=$ & 11) $\frac{8}{23}+\frac{4}{23}-\frac{7}{23}=$ & 12) $\frac{23}{7}-\frac{10}{7}-\frac{50}{7}=$ \\
\hline 13) $\frac{15}{6}+\frac{17}{6}=$ & 14) $\frac{97}{12}+\frac{18}{12}=$ & 15) $\frac{-8}{2}+\frac{54}{2}-\frac{7}{2}=$ & 16) $\frac{43}{15}+\frac{11}{15}-\frac{27}{15}=$ \\
\hline
\end{tabular}

Fuente. Elaboración propia

- $\quad$ Suma de fracciones que tienen diferentes denominadores, pero el mayor es múltiplo del menor:

$$
\frac{1}{2}+\frac{2}{4}=
$$

Al respecto, estas no se pueden sumar directamente; se tienen que expresar en fracciones equivalentes.

1) Observar al menor de los denominadores (en este caso es el número dos), ¿es divisor del otro del otro denominador? La respuesta es afirmativa, entonces:

2) El denominador menor divide al denominador mayor. El resultado (cociente) multiplica a sus dos elementos: numerador y denominador.

3) La fracción obtenida es una equivalente a $\frac{1}{2}=\frac{2}{4}$

4) Las fracciones equivalentes se suman, tomando en cuenta solamente los numeradores.

Ya como fracciones equivalentes se puede realizar la suma de la siguiente manera: 


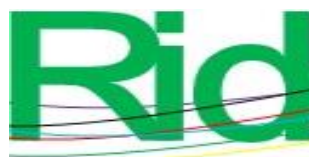

$$
\frac{2}{4}+\frac{2}{4}=\frac{4}{4}=1
$$

Cabe señalar que para el caso de la resta se hace de manera similar.

- $\quad$ Suma de fracciones que tienen diferentes denominadores, pero el mayor no es múltiplo del menor:

$$
\frac{1}{3}+\frac{2}{4}=
$$

Lo siguiente es más general y se puede utilizar para cualquier tipo de fracción común, según Mora (2004).

1) Se hace una multiplicación cruzada de tal manera que se obtienen fracciones equivalentes.

2) Se suman las fracciones:

$$
\frac{1}{3}+\frac{2}{4}=\text { queda de la siguiente manera: } \frac{4}{12}+\frac{6}{12}=\frac{\mathbf{1 0}}{\mathbf{1 2}}
$$

Esto se hace también con las restas.

Ahora bien, ¿cómo saber si un número es divisible por otro? A esta pregunta se le responde con los criterios de divisibilidad, es decir, aquellas normas que dicen cuando un determinado número es divisible por otro porque cumple determinados criterios.

\section{Criterio de divisibilidad del número dos. ¿A qué números divide el dos?}

Encierra en un círculo al número dos y señala qué números son divisibles por ese número de la siguiente tabla 
Tabla 9. Números divisibles por el número dos

\begin{tabular}{|c|c|c|c|c|c|c|c|c|c|}
\hline 1 & 2 & 3 & 4 & 5 & 6 & 7 & 8 & 9 & 10 \\
\hline 11 & 12 & 13 & 14 & 15 & 16 & 17 & 18 & 19 & 20 \\
\hline 21 & 22 & 23 & 24 & 25 & 26 & 27 & 28 & 29 & 30 \\
\hline 31 & 32 & 33 & 34 & 35 & 36 & 37 & 38 & 39 & 40 \\
\hline 41 & 42 & 43 & 44 & 45 & 46 & 47 & 48 & 49 & 50 \\
\hline 51 & 52 & 53 & 54 & 55 & 56 & 57 & 58 & 59 & 60 \\
\hline 61 & 62 & 63 & 64 & 65 & 66 & 67 & 68 & 69 & 70 \\
\hline 71 & 72 & 73 & 74 & 75 & 76 & 77 & 78 & 79 & 80 \\
\hline 81 & 82 & 83 & 84 & 85 & 86 & 87 & 88 & 89 & 90 \\
\hline 91 & 92 & 93 & 94 & 95 & 96 & 97 & 98 & 99 & 100 \\
\hline
\end{tabular}

Fuente: Elaboración propia

- $\quad$ Escriba los números que son divisibles por el número dos:

- De acuerdo con los números escritos, en qué números terminan:

- ¿Si los números aumentaran creen que el comportamiento sería el mismo?

- $\quad$ En términos generales, mencione qué números divide el número dos:

\section{Criterio de divisibilidad del número tres. ¿A qué números divide el tres?}

Tabla 10. Para identificar los números divisibles por el número tres

\begin{tabular}{|l|l|l|l|l|l|l|}
\hline \multicolumn{5}{|c|}{ Múltiplos de tres } & Observaciones por renglón \\
\hline 3 & 12 & 21 & 30 & 39 & 48 & \\
\hline 6 & 15 & 24 & 33 & 42 & 51 & \\
\hline 9 & 18 & 27 & 36 & 45 & 54 & \\
\hline
\end{tabular}

Fuente: Elaboración propia

- ¿Cuánto suman los dígitos en el primer renglón?

- ¿Cuánto suman los dígitos en el segundo renglón?

- ¿Cuánto suman los dígitos en el tercer renglón?

- ¿Si los números aumentaran creen que el comportamiento sería el mismo?

- $\quad$ En términos generales, mencione qué números divide el número tres 
Criterio de divisibilidad del número cinco. ¿A qué números divide el cinco?

Tabla 11. Números del 1 al 100

\begin{tabular}{|c|c|c|c|c|c|c|c|c|c|}
\hline $\mathbf{1}$ & $\mathbf{2}$ & $\mathbf{3}$ & $\mathbf{4}$ & $\mathbf{5}$ & $\mathbf{6}$ & $\mathbf{7}$ & $\mathbf{8}$ & $\mathbf{9}$ & $\mathbf{1 0}$ \\
\hline $\mathbf{1 1}$ & 12 & 13 & 14 & 15 & 16 & 17 & 18 & 19 & 20 \\
\hline $\mathbf{2 1}$ & 22 & 23 & 24 & 25 & 26 & 27 & 28 & 29 & 30 \\
\hline $\mathbf{3 1}$ & 32 & 33 & 34 & 35 & 36 & 37 & 38 & 39 & 40 \\
\hline $\mathbf{4 1}$ & 42 & 43 & 44 & 45 & 46 & 47 & 48 & 49 & 50 \\
\hline $\mathbf{5 1}$ & 52 & 53 & 54 & 55 & 56 & 57 & 58 & 59 & 60 \\
\hline $\mathbf{6 1}$ & 62 & 63 & 64 & 65 & 66 & 67 & 68 & 69 & 70 \\
\hline $\mathbf{7 1}$ & 72 & 73 & 74 & 75 & 76 & 77 & 78 & 79 & 80 \\
\hline $\mathbf{8 1}$ & 82 & 83 & 84 & 85 & 86 & 87 & 88 & 89 & 90 \\
\hline $\mathbf{9 1}$ & 92 & 93 & 94 & 95 & 96 & 97 & 98 & 99 & 100 \\
\hline
\end{tabular}

Fuente: Elaboración propia

Conteste las siguientes preguntas

- Tomando en cuenta los primeros 100 números naturales, ¿qué números divide el cinco?

- ¿Cuál es el criterio de divisibilidad del número cinco?

- ¿Sabes cuáles son los números primos?

- Consulta la biografía de Eratóstenes en la siguiente dirección electrónica: https://es.wikipedia.org/w/index.php?title=Erat\%C3\%B3stenes\&oldid=123632570

No hay una regla (fórmula) que permita obtenerlos. De acuerdo con Laracos (2 de abril de 2011), se describe el procedimiento para aplicarse en la tabla 9. 
Tabla 12. Números del 1 al 100

\begin{tabular}{|c|c|c|c|c|c|c|c|c|c|}
\hline 1 & 2 & 3 & 4 & 5 & 6 & 7 & 8 & 9 & 10 \\
\hline 11 & 12 & 13 & 14 & 15 & 16 & 17 & 18 & 19 & 20 \\
\hline 21 & 22 & 23 & 24 & 25 & 26 & 27 & 28 & 29 & 30 \\
\hline 31 & 32 & 33 & 34 & 35 & 36 & 37 & 38 & 39 & 40 \\
\hline 41 & 42 & 43 & 44 & 45 & 46 & 47 & 48 & 49 & 50 \\
\hline 51 & 52 & 53 & 54 & 55 & 56 & 57 & 58 & 59 & 60 \\
\hline 61 & 62 & 63 & 64 & 65 & 66 & 67 & 68 & 69 & 70 \\
\hline 71 & 72 & 73 & 74 & 75 & 76 & 77 & 78 & 79 & 80 \\
\hline 81 & 82 & 83 & 84 & 85 & 86 & 87 & 88 & 89 & 90 \\
\hline 91 & 92 & 93 & 94 & 95 & 96 & 97 & 98 & 99 & 100 \\
\hline
\end{tabular}

Fuente: Elaboración propia

Procedimiento para encontrar los números primos menores de 100

a) El número uno no se toma en cuenta, a este se le llama número unitario.

b) Encierre el número dos y tache todos sus múltiplos.

c) Encierre el número tres y tache todos sus múltiplos.

d) Encierre el número cinco y tache todos sus múltiplos.

e) Encierre el número siete y tache todos sus múltiplos.

f) Encierre en un círculo los números que no fueron tachados.

g) Escriba todos los números que fueron encerrados en un círculo.

\section{Teorema fundamental de la aritmética}

Dice que todo entero natural no nulo se puede descomponer como un producto de factores primos de forma única.

Cabe señalar que para la siguiente actividad se retoma la información del video de Laracos Math (29 de enero de 2014).

\section{Descomposición factorial de números compuestos}

Factorice el número 30, utilizando a los números primos 
Tabla 13. Factorización

\begin{tabular}{|c|c|}
\hline 30 & 2 \\
\hline 15 & 3 \\
\hline 5 & 5 \\
\hline 1 & \\
\hline
\end{tabular}

Fuente: Elaboración propia

Entonces: $30=2 \times 3 \times 5$

Factorice los siguientes números compuestos

Tabla 14. Factorización de números compuestos

\begin{tabular}{|l|l|l|}
\hline $90=$ & $80=$ & $230=$ \\
\hline $78=$ & $45=$ & $120=$ \\
\hline $56=$ & $74=$ & $320=$ \\
\hline
\end{tabular}

Fuente: Elaboración propia

\section{Simplificación de fracciones}

Significa obtener una fracción irreducible a través de dividir al numerador y denominador por un mismo número.

Simplifica las siguientes fracciones

Tabla 15. Fracciones comunes

\begin{tabular}{|l|l|l|}
\hline$\frac{150}{130}=$ & $\frac{130}{140}=$ & $\frac{200}{300}=$ \\
\hline$\frac{600}{580}=$ & $\frac{656}{532}=$ & $\frac{180}{200}=$ \\
\hline$\frac{1000}{800}=$ & $\frac{900}{880}=$ & $\frac{1500}{2800}=$ \\
\hline$\frac{2600}{3800}=$ & $\frac{1500}{1280}=$ & $\frac{1900}{1600}=$ \\
\hline
\end{tabular}

Fuente: Elaboración propia 


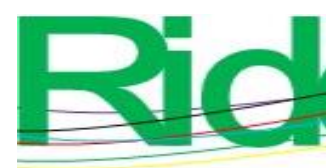

Revista Iberoamericana para la Investigación y el Desarrollo Educativo ISSN $2007-7467$

\section{El mínimo común múltiplo}

El mínimo común múltiplo $(\mathrm{mcm})$ es el menor común múltiplo de una cierta cantidad de números (véase tabla 13).

Tabla 16. Números y múltiplos

\begin{tabular}{|c|c|}
\hline Números & Múltiplos \\
\hline 4 & $4,8,12,16,20,24,28,32,36,40,44,48,52,56,60,64,68,72,76,80,84 \ldots$ \\
\hline 6 & $6,12,18,24,30,36,42,48,54,60,66,72,78,84,90,96, \mathbf{1 0 2}, 108,114 \ldots$ \\
\hline
\end{tabular}

\section{Fuente: Elaboración propia}

- ¿Cuáles son los múltiplos comunes de cuatro y seis?

- ¿Cuál es el menor común múltiplo de ellos?

A este número se le conoce como el $\mathrm{mcm}$. Existen otros procedimientos para calcular el mcm, como la descomposición en factores primos.

Calcular el mínimo múltiplo de los siguientes números

a) $\operatorname{mcm}(12,24,14)=$

b) $\operatorname{mcm}(3,4,5)=$

Se escriben los números que están dentro de cada paréntesis para descomponerlos en factores, utilizando solamente números primos. En este caso, importa demasiado que en cada división el que resuelve se pregunte si alguno de los números que observa es divisible por el primer número primo; si no fuera posible, se continúa con los demás números primos mayores a dos. 


\section{Revista Iberoamericana para la Investigación y el Desarrollo Educativo ISSN $2007-7467$}

Tabla 17. Descomposición factorial del inciso $a$ )

\begin{tabular}{|l|l|}
\hline $12,24,14$ & 2 \\
\hline $6,12,7$ & 2 \\
\hline $3,6,7$ & 2 \\
\hline $3,3,7$ & 3 \\
\hline $1,1,7$ & 7 \\
\hline $1,1,1$ & \\
\hline
\end{tabular}

Fuente: Elaboración propia

$\operatorname{mcm}(12,24,14)=2 \times 2 \times 2 \times 3 \times 7=168$

Tabla 18. Descomposición factorial del inciso $b$ )

\begin{tabular}{|l|l|}
\hline $3,4,5$ & 2 \\
\hline $3,2,5$ & 2 \\
\hline $3,1,5$ & 3 \\
\hline $1,1,5$ & 5 \\
\hline $1,1,1$ & \\
\hline
\end{tabular}

Fuente: Elaboración propia

$\operatorname{mcm}(3,4,5)=2 \times 2 \times 3 \times 5=60$

Encuentra el mcm de los siguientes números (cópialos en tu cuaderno)

Tabla 19. Números compuestos

\begin{tabular}{|l|l|l|l|}
\hline $\operatorname{mcm}(12,48,32)=$ & $\operatorname{mcm}(24,12,6)=$ & $\operatorname{mcm}(12,24,48)=$ & $\operatorname{mcm}(120,6,30)=$ \\
\hline $\operatorname{mcm}(20,10,5)=$ & $\operatorname{mcm}(10,30,40)=$ & $\operatorname{mcm}(12,48,96)=$ & $\operatorname{mcm}(24,12,120)=$ \\
\hline
\end{tabular}

Fuente: Elaboración propia 


\section{Revista Iberoamericana para la Investigación y el Desarrollo Educativo ISSN $2007-7467$}

\section{Fracciones mixtas}

Existen diferentes métodos. El que se explica aquí se realiza de la siguiente forma: cada fracción mixta se expresa a una impropia (multiplicando el denominador por la parte entera y sumándole el numerador, el cual se escribe como numerador de la fracción común, cuyo denominador será el mismo denominador que posee la parte fraccionaria).

Expresar una fracción mixta a una impropia

$$
\begin{aligned}
& 3 \frac{1}{4}=\frac{13}{4} \\
& 3 \frac{1}{4}-2 \frac{1}{3}-4 \frac{1}{2} \\
& =\frac{13}{4}-\frac{7}{3}-\frac{9}{2}=\frac{39-28-54}{12}=\frac{39-82}{12}=\frac{-43}{12}=-3 \frac{7}{12}
\end{aligned}
$$

Realiza las siguientes sumas en tu cuaderno

Tabla 22. Suma de fracciones mixtas

\begin{tabular}{|l|l|r|r|}
\hline a) $6 \frac{1}{2}+7 \frac{3}{3}+8 \frac{5}{4}=$ & b) $4 \frac{2}{3}+6 \frac{1}{6}+8 \frac{3}{9}=$ & c) $2 \frac{1}{6}+3 \frac{2}{9}=$ & d) $6 \frac{5}{7}+8 \frac{3}{7}+12 \frac{1}{7}=$ \\
\hline e) $6 \frac{4}{9}+4 \frac{1}{3}+5 \frac{7}{6}=$ & f) $5 \frac{2}{3}-4 \frac{1}{4}=$ & g) $\frac{7}{3}+\frac{3}{8}+\frac{12}{4}$ & h) $\frac{3}{5}+7 \frac{5}{8}+3 \frac{2}{3}=$ \\
& &
\end{tabular}

\section{Fuente: Elaboración propia}

\section{Multiplicaciones}

Se multiplica el numerador con numerador y denominador con denominador. Algunos ejemplos se observan en la tabla 20.

Tabla 23. Ejemplos de multiplicación de fracciones

\begin{tabular}{|l|l|l|}
\hline a) $\frac{5}{7} x \frac{3}{2}=\frac{15}{14}$ & b) $\frac{3}{8} x 7=\frac{3}{8} x \frac{7}{1}=\frac{21}{8}$ & c) $(-9) \frac{2}{3}=\left(\frac{-9}{1}\right) \frac{2}{3}=\frac{-18}{3}=-6$ \\
\hline
\end{tabular}

Fuente: Elaboración propia 
Resuelve las siguientes multiplicaciones

Tabla 24. Ejercicios de multiplicaciones de fracciones

\begin{tabular}{|l|l|l|l|}
\hline a) $\frac{5}{9} \times \frac{18}{20}=$ & b) $\frac{1}{5} \times \frac{5}{3}=$ & c) $\frac{50}{25} \times \frac{100}{150}=$ & d) $\frac{14}{18} \times \frac{9}{7}=$ \\
\hline e) $(12)\left(\frac{9}{7}\right)=$ & f) (15) $\left(\frac{-4}{3}\right)=$ & g) (8) $\left(\frac{-37}{3}\right)=$ & h) $\left(\frac{13}{3}\right)(8)=$ \\
\hline
\end{tabular}

Fuente: Elaboración propia

\section{Divisiones}

Estas se multiplican en forma cruzada. Algunos ejemplos se muestran en la tabla 22.

Tabla 25. Ejemplos de divisiones aritméticas
a) $\frac{3}{6} \div \frac{2}{5}=\frac{15}{12}=\frac{5}{4}$
b) $6 \div \frac{2}{3}=\frac{6}{1} \div \frac{2}{3}=\frac{18}{2}=9$
c) $4 \div \frac{2}{9}=\frac{4}{1} \div \frac{2}{9}=\frac{36}{2}=18$

Fuente: Elaboración propia

Resuelve las siguientes divisiones

Tabla 26. Ejercicios de divisiones aritméticas

\begin{tabular}{|l|l|l|}
\hline a) $\frac{24}{10} \div \frac{15}{30}=$ & b) $\frac{5}{3} \div \frac{9}{20}=$ & c) $\frac{18}{36} \div \frac{1}{3}=$ \\
\hline d) $\frac{11}{34} \div \frac{8}{6}=$ & e) $\frac{13}{4} \div \frac{7}{6}=$ & f) $\frac{12}{15} \div \frac{-3}{-4}=$ \\
\hline
\end{tabular}

\section{Fuente: Elaboración propia}

\section{Irracionales}

En este punto se e retoma lo que menciona Scaglia (2000) del conflicto cognitivo para el tratamiento de los números racionales en la recta numérica. 
Figura 13. Números irracionales

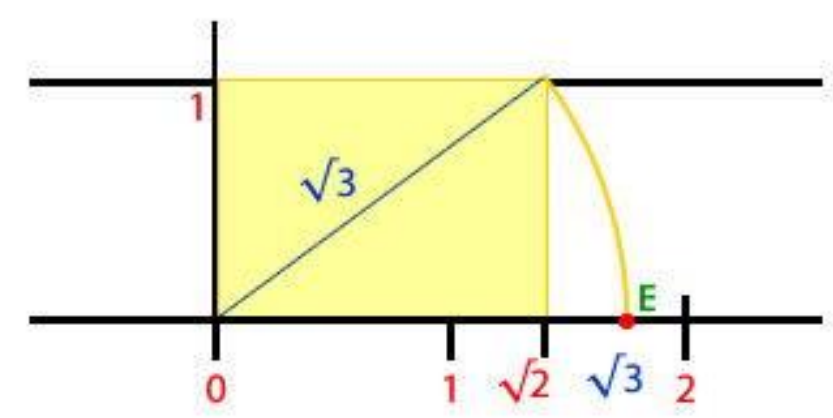

Fuente: https://www.portaleducativo.net/segundo-medio/4/ordenar-numeros-irracionalesrepresentarlos-en-recta-numerica

Hasta la creación de los números racionales, parecía que ya se tenían todos los números y ya no faltaba ninguno. Según Valdez (2008), en tiempo de Pitágoras de Samos, matemático griego que vivió aproximadamente en los años 569 y 475 a. C., la escuela pitagórica planteaba que hasta ahí la numeración llegaba, sin embargo, comenta la historia que dado un problema de cálculo un resultado no pertenecía a los números conocidos.

El problema era el siguiente: "Dado un triángulo rectángulo isósceles de magnitud igual a la unidad, calcular la medida de su hipotenusa”.

Tabla 27. El triángulo rectángulo y el teorema de Pitágoras

\begin{tabular}{|l|c|c|c|}
\hline \multicolumn{1}{|c|}{ Datos } & Fórmula & Sustitución & Análisis \\
\hline El cateto $\mathrm{a}=1$ & $c^{2}=a^{2}+b^{2}$ & $c=\sqrt{1^{2}+1^{2}}$ & El valor de la $\sqrt{2}$ no \\
El cateto $\mathrm{b}=1$ & Despejar a $c$ & $c=\sqrt{1+1}$ & se puede expresar \\
El cateto $\mathrm{c}=$ & $c=\sqrt{a^{2}+b^{2}}$ & $c=\sqrt{2}$ & como una fracción. \\
\hline
\end{tabular}

Fuente: elaboración propia

Reflexión: Entonces, buscando las raíces cuadradas de otros números, nos damos cuenta que sucede algo similar con la $\sqrt{2}$, otro de los números racionales es el valor de $\pi$, que también tiene decimales no periódicos y el valor de Euler, con un valor aproximado de 2.7181 .

En este campo numérico no se llevarán a cabo las operaciones, puesto que corresponde al nivel de medio superior y, además, el tiempo destinado a resolver esta secuencia corresponde a un tiempo fuera del horario escolar. 


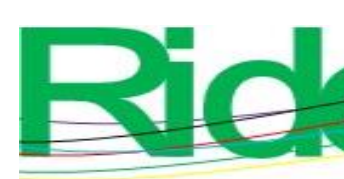

Revista Iberoamericana para la

Investigación y el Desarrollo Educativo

ISSN 2007 - 7467

\section{Resultados de la intervención docente}

Para analizar los resultados se tomaron en cuenta dos planes de estudio que se vinculan con la formación de docentes (Secretaría de Educación Pública [SEP], 2011, 2017a). La secuencia didáctica planteada a los alumnos resultó satisfactoria por la participación de todos los estudiantes, quienes fueron contestando cada una de las preguntas y resolviendo las operaciones.

Por la estructura de la secuencia didáctica, el rol del docente siempre fue de facilitador, mientras los alumnos tuvieron una participación activa y constructivista.

Es importante mencionar que la obra de Baldor (1996) fue importante para contextualizar el trabajo de este artículo. Igualmente lo fue el documento Modelo educativo para la educación obligatoria. Educar para la libertad y la creatividad (SEP, 2017b).

\section{Discusión}

Antes de la aplicación de la secuencia didáctica, había una amplia expectación de curiosidad de parte del alumnado, principalmente por la posible dificultad del tema; sin embargo, cuando fueron recibiendo las hojas de trabajo y leyendo las actividades, se dieron cuenta que no solo producían nuevo conocimiento, sino también reforzaban el que ya tenían, es decir, con esta secuencia sistematizaron su información, pero, además, conocieron de manera general por qué se creaban otros campos numéricos.

La limitación que se encontró en el presente trabajo es la escasa reproducibilidad que puede hacerse en la escuela para determinar algunos otros factores que incidan en el aprendizaje, lo que daría mayor información para el artículo. Si fuera una escuela secundaria, con seis grupos del mismo grado, seguramente se integraría más información al trabajo que se presenta.

El campo numérico donde empezaron a tener problemas los estudiantes fue en los números enteros. Aquí se les precisó que preferentemente, como futuros docentes, no pasaran al siguiente campo porque, si lo descuidaban, tendrían muchas dificultades para resolver las ecuaciones lineales y cuadráticas, puesto que el error de signos ocasionaría que no se pudieran resolver los problemas planteados. 


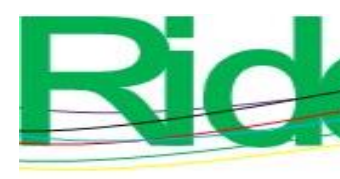

Revista Iberoamericana para la Investigación y el Desarrollo Educativo ISSN 2007 - 7467

En cuanto a los números racionales, siempre ha sido un dolor de cabeza para aprenderlos, pero ahora, a pesar de algunas dificultades, tenían un mejor apoyo con el conocimiento previo de los criterios de divisibilidad, números primos y la factorización de los números compuestos.

Como fortaleza del trabajo de investigación se observó la amplia disposición del alumnado para realizar las actividades propuestas, así como la sistematización del trabajo para que las primeras fueran sustento de las siguientes, esto significó que, al finalizar el trabajo académico, sus ideas numéricas estuvieran mejor organizadas.

En cuanto al área de debilidades, se puede mencionar que los futuros profesores de las escuelas telesecundarias en varias ocasiones abordan contenidos del nivel de manera superficial, sin que ello demerite la calidad educativa. Es por eso por lo que los profesores formadores de docentes tienen que realizar trabajos sustanciosos en espacios pequeños de tiempo, con la finalidad de ser productivos.

\section{Conclusiones}

El trabajo realizado con los estudiantes de la licenciatura en Enseñanza y Aprendizaje en Telesecundaria fue provechoso para su formación profesional, puesto que, a partir de ahora, en cada intervención matemática, sabrán de qué campo numérico están hablando y qué reglas se deben de aplicar adecuadamente. Esto es de suma importancia para la resolución de problemas.

Los profesores que no conocen la clasificación de los números reales tienden a equivocarse en las operaciones matemáticas, por eso es indispensable que conozcan las reglas que se establecen en cada campo numérico.

Los estudiantes lograron diferenciar los diversos campos numéricos. Ahora saben cómo están organizados los números reales. Que en cada campo existe una regla que rige las operaciones que ahí se realizan, por ejemplo, no es lo mismo $7-2$, que $2-7$; para el primer caso, el resultado es +5 y para el segundo es -5 , dichos resultados representan cosas diferentes, a saber, en el primero, si se hablara de unidades monetarias, significa que cierta persona posee cinco pesos, mientras que en el segundo caso, el significado es que la persona tiene un adeudo de cinco pesos. 

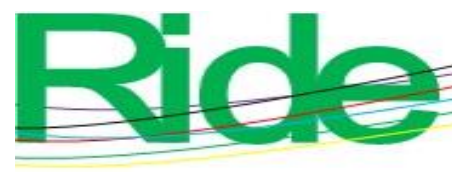

Revista Iberoamericana para la Investigación y el Desarrollo Educativo

ISSN $2007-7467$

Ahora el alumno normalista tiene más registros para realizar operaciones, no solo el numérico y verbal, también el gráfico, que en este caso se representa en una recta numérica, la cual apoya especialmente a la suma y resta de números con signo.

Establece una clasificación de las fracciones de acuerdo con el denominador que posee; sabe que si el denominador es menor que la unidad, esta se llama fracción propia, y si es igual o mayor que la unidad se le conoce como fracción impropia.

La construcción de los criterios de divisibilidad se dio con los siguientes números: dos, tres y cinco, haciendo operaciones, observaciones y conjeturas sobre la división de los números antes mencionados.

Con las acciones anteriores, se identificaron los números primos (los que solo tienen dos divisores) menores que 100 y se les notificó que, hasta la actualidad, no hay ninguna fórmula que ayude a calcularlos; existe un procedimiento que fue diseñado por Eratóstenes, quien fue un matemático griego que murió en 194 a. C. y que uno de sus cálculos más reconocidos es haber mencionado la longitud de la circunferencia de la Tierra.

Con el uso de los números primos, los compuestos (aquellos que tienen más de dos divisores) se pueden descomponer en factores primos; dicha acción facilita las operaciones de fracciones de suma y resta.

Como se observa, la secuencia didáctica consistió en encadenar situaciones que permitieron lograr sentar las bases de la aritmética para los estudiantes de la Escuela Normal Urbana Federal Prof. Rafael Ramírez. 


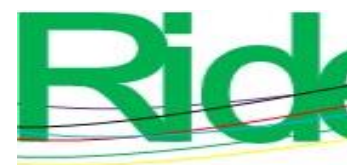

Revista Iberoamericana para la Investigación y el Desarrollo Educativo ISSN 2007-7467

\section{Referencias}

Baldor, A. (1996). Aritmética. México: Publicaciones Cultural.

Carreon, D. (24 de julio de 2016). Suma de fracciones con diferente denominador. (video de YouTube). https://www.youtube.com/watch?v=LVHo5xvsvO0. Recuperado de https://www.youtube.com/watch?v=LVHo5xvsvO0.

Céspedes, E. (27 de septiembre de 2017). Balanza comercial. ABCfinanzas. Recuperado de https://www.abcfinanzas.com/principios-de-economia/que-es-la-balanza-comercial.

Laracos Math. (2 de abril de 2011). Números primos con la criba de Eratóstenes. (video de YouTube). Recuperado de https://www.youtube.com/watch?v=XytXXaKytVU.

Laracos Math. (29 de enenero de 2014). Factorizacion de numeros a factores primos. (video de YouTube). Recuperado de https://www.youtube.com/watch?v=Yul9zO2ONdQ.

MateFacil. (8 de agosto de 2016). Clasificación de números: naturales, enteros, racionales, irracionales, reales, complejos. (video de YouTube). Recuperado de https://www.youtube.com/watch?v=rtNC7g1h_JA.

Matemáticas profe Alex. (28 de octubre de 2018). Comprendiendo la suma y resta de fracciones | Explicación completa. (video de YouTube). Recuperado de https://www.youtube.com/watch?v=YpSb9LlsFv8.

Méndez, Z. (1993). Aprendizaje y cognición. Barcelona, España: Universidad Estatal a Distancia.

Mora, L. (2004). Concepciones de estudiantes de licenciatura en matemáticas sobre números reales. (tesis de maestría). Universidad Pedagógica Nacional, Bogotá.

Rodríguez, E. (s. f.). Juegos con fracciones: lectura, comparación, fracciones. (juego educativo). Recuperado de https://www.tes.com/lessons/zABJh2K_GmWTdg/fracciones.

Sáenz, E. (22 de abril de 2019). ¿Qué son realmente los números reales? (video de YouTube). Universitat Politècnica de València, España. Recuperado de https://www.youtube.com/watch?v=xOjQ3u7jSLQ.

Sánchez, D. (2 de febrero de 2014). Las matemáticas en la prehistoria $5^{\mathrm{a}}$ parte: Los huesos de Lebombo y de Ishango. Prehistoria al Día. Recuperado de https://prehistorialdia.blogspot.com/2014/02/las-matematicas-en-la-prehistoria5.html. 

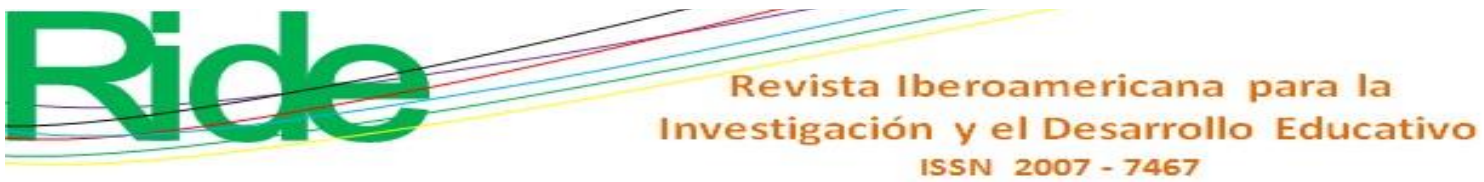

Scaglia, S. (2000). Dos conflictos al representar números reales. (tesis doctoral). Universidad de Granada, España.

Secretaría de Educación Pública [SEP]. (2011). Programas de Estudio 2011. Guía para el maestro. Educación básica secundaria. México: CONALITEG.

Secretaría de Educación Pública [SEP]. (2017a). Aprendizajes clave para la educación integral. Plan y programas de estudio para la educación básica. México: Secretaría de Educación Pública.

Secretaría de Educación Pública [SEP]. (2017b). Modelo educativo para la educación obligatoria. Educar para la libertad y la creatividad. México: Secretaría de Educación Pública.

Struik, D. (1998). Historia concisa de las matemáticas. México: Instituto Politécnico Nacional.

Tu profe en línea. (13 de mayo de 2015). Clasificación de Fracciones - Completo. (video de YouTube). Recuperado de https://www.youtube.com/watch?v=2qqoiCXF6YI.

Valdez, V. (2008). Los conjuntos numéricos a través de la historia. (tesina). Instituto Superior de Fundación Suzuki, Argentina.

Wikipedia. (30 de abril de 2018). Eratóstenes. Recuperado de https://es. wikipedia.org/w/index.php?title=Erat\%C3\%B3stenes\&oldid=123632570.

Wikipedia. (22 de abril de 2019). Número real. Recuperado de https://es.wikipedia.org/w/index.php?title=N\%C3\%BAmero_real\&oldid=12383544 7.

Wikipedia. (26 de noviembre de 2019). Papio. Recuperado de https://es.wikipedia.org/wiki/Papio\#/media/File:Olivebaboon.jpg. 